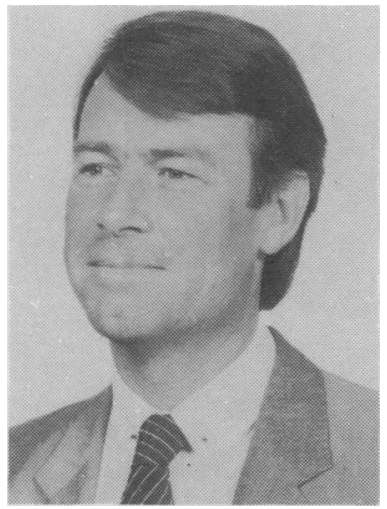

\title{
INJURIES TO THE LATERAL LIGAMENT OF THE ANKLE: ASSESSMENT AND TREATMENT
}

M. A. HUTSON and J. P. JACKSON, FRCS

Sports Injury Clinic, General Hospital, Nottingham, UK

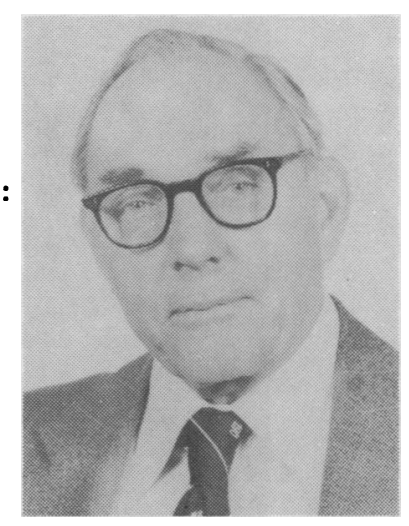

ABSTRACT

Initial assessment of severity of injury to the lateral ligament of the ankle is by clinical examination. If it is thought that there is a possibility of either lateral or anterior instability, patients are investigated radiologically by anterior and inversion stress films.

If instability is demonstrated a peroneal tenogram is carried out to exclude damage to the calcaneo-fibular ligament.

Treatment is by means of a cast brace if instability is present. The advantages of this are preservation of calf power and improved joint function.

The early results are reported in relation to the improvement in stability and early return to sporting activity.

Key words: Instability - lateral and anterior. Stress X-rays. Peroneal tenogram. Cast brace.

\section{INTRODUCTION}

There is now substantial evidence that the diagnosis of lateral ligament rupture of the ankle requires the use of lateral stress $\mathrm{X}$-rays to demonstrate anterior subluxation of the talus from the ankle mortice in addition to AP stress $X$-rays that reveal varus tilt (Hackenbruch et al, 1979; Glasgow et al, 1980; Lindstrand, 1976), seen in Figs. 2 and 3. Lateral stress films are more reliable in revealing anterior talo-fibular ligament rupture, Figs. 4 and 5. The addition of the peroneal tenogram to routine stress films results in the ability to diagnose rupture of the calcaneo-fibular ligament because of the intimate association of the latter ligament with the sheath of the peroneal tendons, Figs. 6 and 7. Thus Evans (1979) has demonstrated the usefulness of the stress-tenogram in investigation of injuries to the lateral ligament of the ankle, and has confirmed the degree of diagnostic accuracy at operative repair in a group of patients. Such precision of diagnosis of structural damage is essential for a realistic assessment of the place of the various methods available in the management of lateral ligamentous rupture. Standard methods of treat-

Communications to:

Dr. M. A. Hutson

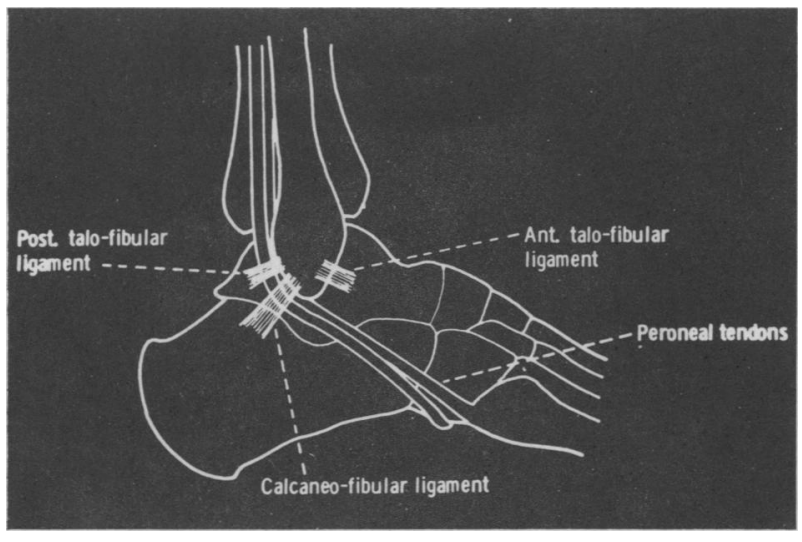

Fig. 1: Anatomical representation of lateral ligament complex of ankle joint.

ment are the conservative approach (using plaster of Paris) and the surgical reconstructive approach, both of which necessitate periods of immobilisation. An alternative line of treatment uses a cast brace made of Scotchcast and plastic heel cup in which a restricted degree of plantar flexion of the ankle is allowed during maintenance of a reasonably normal gait. Work now 


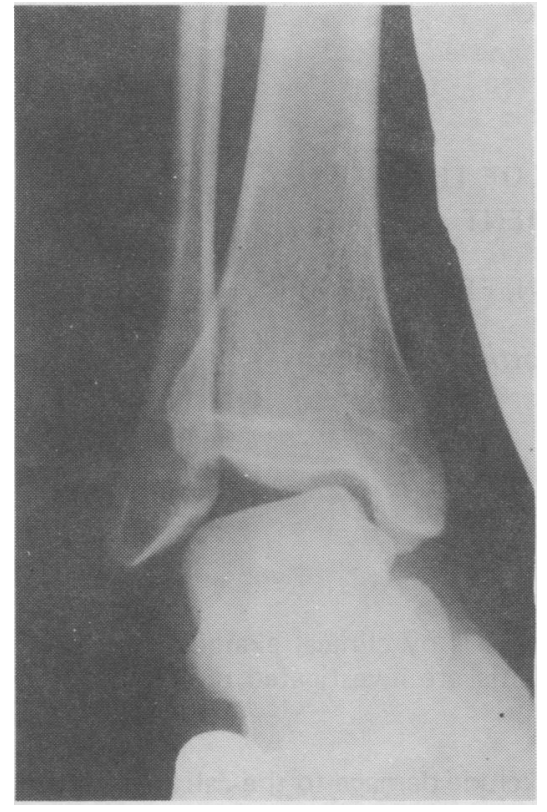

Fig. 2: Stress $X$-ray of ankle showing varus instability (right ankle).

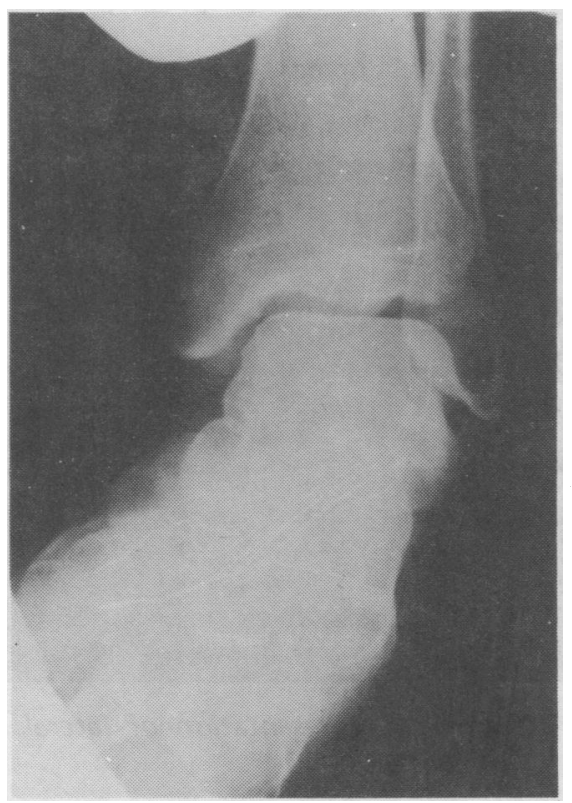

Fig. 3: Stress $X$-ray of other (normal) ankle for comparison (left ankle).

being carried out (Hutson and Jackson, Nottingham Sports Injury Clinic) compares the clinical assessment of severity of lateral ligament injury with the radiological

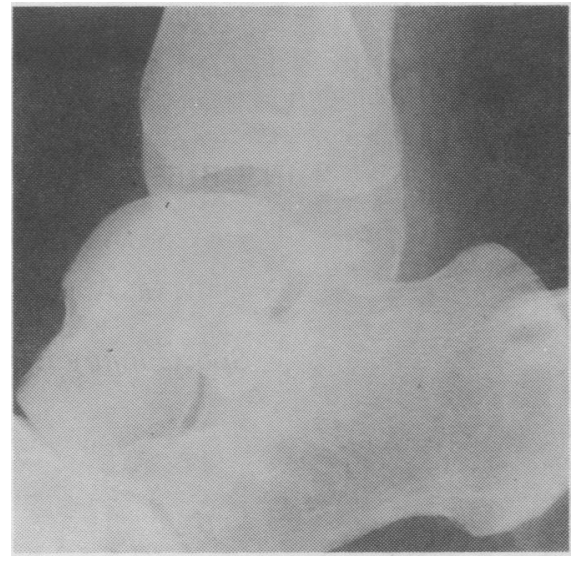

Fig. 4: Stress $X$-ray of ankle revealing anterior instability (right ankle).

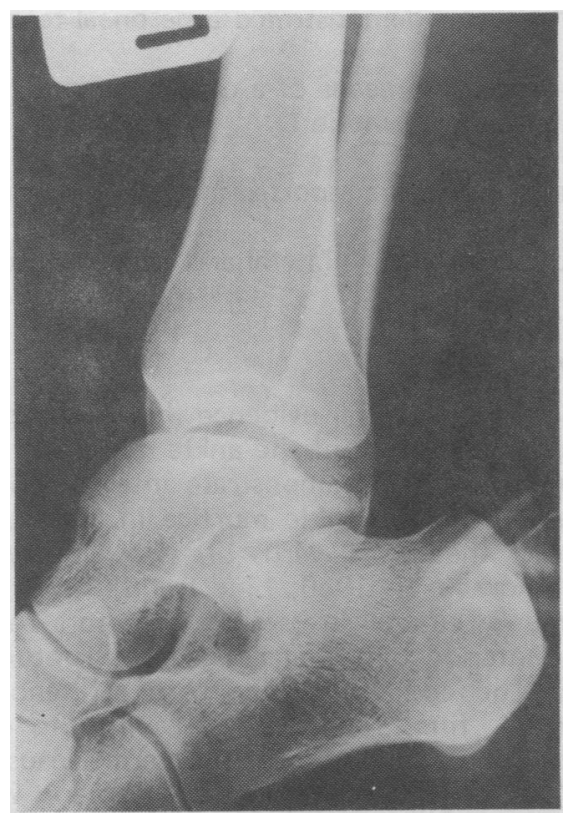

Fig. 5: Normal stress $X$-ray (other ankle) for comparison (left ankle).

assessment using the stress tenogram; the results of treatment using the cast brace are assessed for both uniligamentous (anterior talo-fibular ligament) and biligamentous (anterior talo-fibular and clacaneo-fibular ligaments) injuries by further follow-up that includes clinical and radiological assessment for stability at three months. 


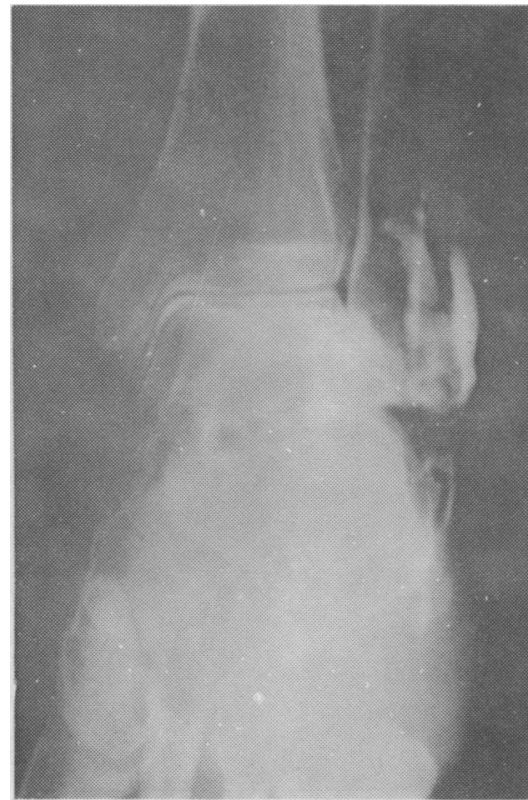

Fig. 6: Peroneal tenogram showing presence of dye in ankle joint.

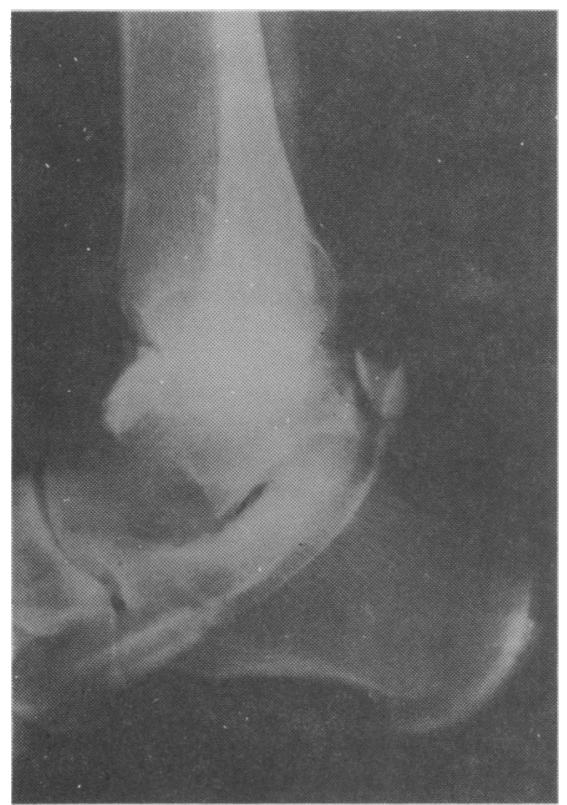

Fig. 7: Peroneal tenogram showing presence of dye in and anterior to ankle joint.

\section{Aetiology}

Lateral ligamentous injuries usually result from a combination of plantar flexion and inversion of the ankle. The anterior talo-fibular ligament is initially damaged, and with increasing inversion the calcaneofibular and then the complete lateral ligamentous complex. Occasionally pure adductive force with the ankle plantigrade may give rise to an isolated rupture of the calcaneo-fibular ligament. Patients presenting to the Accident and Emergency Department, University Hospital, Nottingham are referred to the Sports Injury Clinic at Nottingham General Hospital if sports-related and are seen in the Sports Injury Clinic within three to four days (clinics are held on Mondays and Thursdays).

\section{Clinical Assessment}

Clinical assessment includes the anterior drawer test for anterior instability: with the foot slightly plantar flexed the talus emerges anteriorly from the mortice as a result of forward pressure behind the heel, and inverts. This movement can be seen and felt without the injection of local anaesthetic. In this respect it differs from the assessment of lateral instability by passive inversion that usually requires anaesthesia in some form for patients' relaxation in acute injuries. Following suspicion or demonstration of instability further assessment is made.

\section{Radiological Assessment}

The patient subsequently receives an injection of local anaesthetic to the affected area prior to having inversion and anterior stress films performed. A $4 \mathrm{~kg}$ sandbag is applied to the anterior aspect of the lower tibia with the heel resting on a plinth during the anterior stress test and lateral films taken two minutes after application of stress. Conventional inversion stress films are taken following manual passive inversion of the injured ankle. On inversion stress, talar tilt over $10^{\circ}$ is taken as probably abnormal. More reliance though is placed on the anterior stress films in which $6 \mathrm{~mm}$ from the posterior articular surface of the tibia to the nearest point of the talus is considered abnormal; this distance is often 8 to $10 \mathrm{cms}$ in the more severe injury.

The results of stress examinations are as follows:

1. Ankle stable, both clinically and on stress testing.

2. Anterior stress positive. Varus stress negative.

3. Anterior stress positive. Varus stress positive.

4. Anterior stress negative. Varus stress positive.

Tenograms are performed in groups 2,3 and 4 . Patients with positive anterior stress (groups 2 and 3) who have a positive tenogram are thus diagnosed as having biligamentous injury (indeed the presence of dye posteriorly also indicates complete joint disruption). A negative tenogram indicates anterior talo-fibular ligament rupture only. 
Patients in group 4 may have a false positive varus stress test, a false negative anterior stress test or a calcaneo-fibular rupture in isolation; a tenogram will be positive in the latter group. Patients may have suffered previous ligamentous injury of similar nature and may therefore have some degree of initial ligamentous laxity - further injury really categorises them as acute on chronic sufferers and account must be taken of this when assessing the results of stress tests. Stress tests of the non-injured ankle may be useful for comparison, though variation of norms of talar tilt on a varus stress are widely recognised and may occur in the same individual. Hackenbruch suggests however that a reliable assessment of anterior subluxation may be made when the tibial-talar distance on lateral $X$-rays is greater than $3 \mathrm{~mm}$ on comparing the two sides. Further consideration must be given to the fact that in the group of mainly young people, in whom these injuries occur, previous injury to the opposite "normal" ankle may well have been sustained, and it is not at all unusual to demonstrate bilateral positive stress tests due to ligamentous laxity. The subsequent tenogram should then help to diagnose the severity of the recent injury.

\section{Management}

Patients who do not demonstrate ligamentous laxity, either clinically or radiologically, are actively mobilised without support.

Patients with demonstrable instability, either uniligamentous or biligamentous, are treated for an initial period of three weeks in a cast brace that comprises plastic ankle hinges and heel cup attached to a Scotchcast below knee cylinder terminating immediately above the ankle. Effective lateral support is thereby given to the ankle and subtalar joints whilst a restricted degree of plantar flexion allows mobilisation with as near normal a heel-toe gait as possible. Patients almost invariably feel comfortable immediately following application, and ambulation is neither difficult nor painful. Foot hygiene is maintained by patients' ability to detach and re-attach with ease the ankle hinges and heel cup from the Scotchcast cylinder, Figs. 8 and 9.

Besides patient comfort the advantages of this method of treatment stem principally from the degree of permitted mobilisation. Salter (1975) has demonstrated improvement in nutrition of articular cartilage when joints are mobilised. Less muscle wasting (particularly important in the calf) and joint stiffness occur, both important factors in the subsequent rehabilitation of trained athletes. After three weeks the brace is removed and active physiotherapeutic rehabilitation is continued. It has been found possible, for instance in two international soccer players, for return to match fitness in five to six weeks from date of injury. Subsequent stress $X$-rays in both these players have shown reduction of instability, Fig. 10 . Such rapid rehabili-

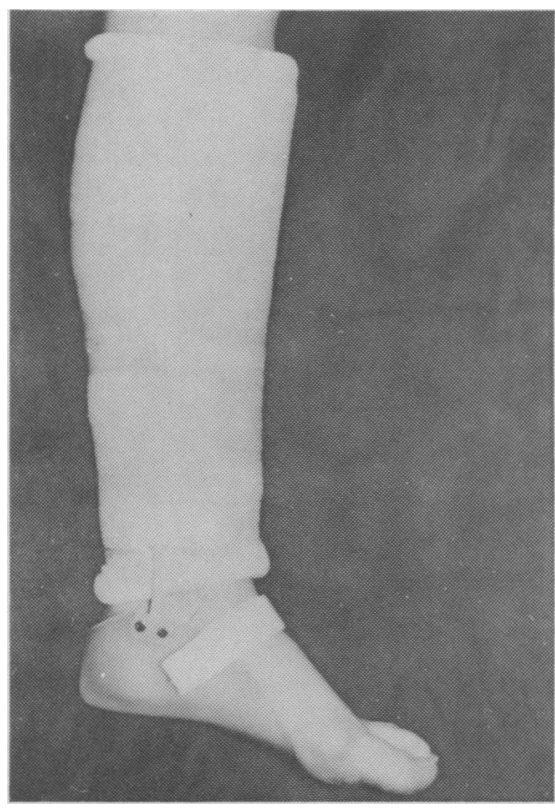

Fig. 8: Cast brace for ankle.

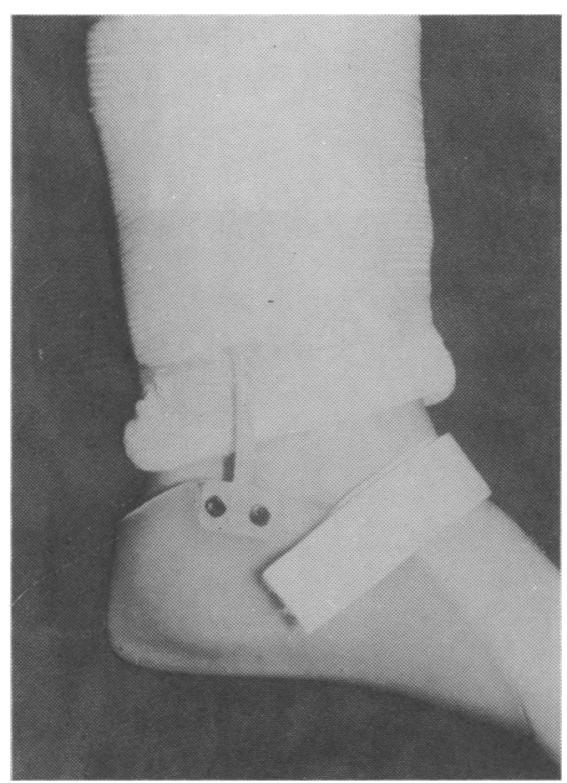

Fig. 9: Cast brace.

tation is impossible using either a surgical or plaster immobilisation approach. We have a strong impression, following initial assessment and three month follow-up on 30 patients, that clinical stability can be achieved using a cast brace (so far subsequent functional ankle 


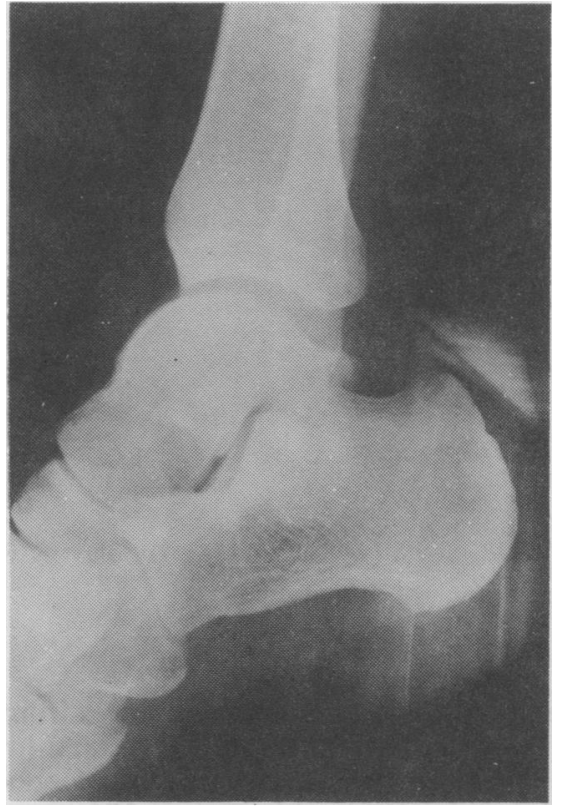

instability has not been detected) and follow-up films have shown considerable diminution in the degree of excessive talar movement on stress tests.

Further assessment should be made by comparison with management of similar cases by surgical and more conservative below knee plaster of Paris and this is being undertaken.

Fig. 10: Stress film of injured ankle demonstrating recovery (no instability) (right ank/e).

\section{REFERENCES}

Glasgow, M., Jackson, A. and Jamieson, A., 1980 "Instability of the ankle after injury to the lateral ligament". Journal of Bone and Joint Vol. 62B: 196-200.

Evans, A. G. and Frenyo, S. D., 1979 "The stress tenogram in the diagnosis of ruptures of the lateral ligament of the ankle". Journal of Bone and Joint (Br.) 61: 347-351.

Hackenbruch, W., Noesberger, B. and Debrunner, H. U., 1979 "Differential diagnosis of ruptures of the lateral ligaments of the ankle joint". Arch.Orthop Trauma Surg. 93: 293-301.

Lindstrand, A. and Mortensson, W., 1977 "Anterior instability in the ankle joint following acute lateral sprain". Acta Radiological Diagnosis 18: 529-538.

Salter, R. B., Simmonds, D. F., Malcolm, B. W., Rumble, E. J. and MacMichael, D., 1975 "The effects of continuous passive motion on the healing of articular cartilage defects". Journal of Bone and Joint (A) 57: 570. 